\title{
Assessment of Patient-Reported Outcomes After Polytrauma: A Systematic Review
}

\author{
Michaela Ritschel ( $\nabla$ michaela.ritschel@hhu.de) \\ Institute for Health Services Research and Health Economics https://orcid.org/0000-0001-8686-7956 \\ Silke Kuske \\ Fliedner Fachhochschule Dusseldorf, University of Applied Sciences \\ Imela Gnass \\ Paracelsus Medical University, Institute of Nursing Science and Practice, Salzburg, Austria
}

\section{Silke Andrich}

Institute for Health Services Research and Health Economics, Centre for Health and Society, Faculty of Medicine, Heinrich-Heine-University Düsseldorf, Düsseldorf, Germany

\section{Kai Moschinski}

Institute for Health Services Research and Health Economics, Centre for Health and Society, Faculty of Medicine, Heinrich-Heine-University Düsseldorf, Düsseldorf, Germany

\section{Sandra Olivia Borgmann}

Institute for Health Services Research and Health Economics, Centre for Health and Society, Faculty of Medicine, Heinrich-Heine-University Düsseldorf, Düsseldorf, Germany

\section{Annegret Hermann-Frank}

Institute for Health Services Research and Health Economics, Centre for Health and Society, Faculty of Medicine, Heinrich-Heine-University Düsseldorf, Düsseldorf, Germany

\section{Maria-Inti Metzendorf}

Cochrane Metabolic and Endocrine Disorders Group, Institute of General Practice, Faculty of Medicine, Heinrich-Heine-University Düsseldorf, Düsseldorf, Germany

\section{Charlotte Wittgens}

Institute for Health Services Research and Health Economics, Centre for Health and Society, Faculty of Medicine, Heinrich-Heine-University Düsseldorf,

Düsseldorf, Germany

\section{Sascha Flohé}

Department of Trauma, Orthopaedics and Hand Surgery, Städt. Klinikum Solingen, Solingen, Germany

Johannes Sturm

AUC-Academy for Trauma Surgery GmbH, München, Germany

\section{Joachim Windolf}

Department of Orthopaedics and Traumatology, University Hospital Düsseldorf, Germany

\section{Andrea Icks}

Institute for Health Services Research and Health Economics, Centre for Health and Society, Faculty of Medicine, Heinrich-Heine-University Düsseldorf, Düsseldorf, Germany

\section{Research}

Keywords: Patient-reported outcomes, quality of life, polytrauma, multiple trauma, activities of daily living, social participation, systematic review, instruments Posted Date: October 19th, 2020

DOl: https://doi.org/10.21203/rs.3.rs-92709/v1

License: (c) (i) This work is licensed under a Creative Commons Attribution 4.0 International License. Read Full License 


\section{Abstract}

Background: Tools assessing patient-reported outcomes (PROs) after polytrauma have not been systematically reviewed so far. Therefore, we sought to: (1) collect instruments that assess health-related quality of life (HRQoL), activities of daily living (ADL), and social participation during follow-up after polytrauma, (2) describe their use (e.g. frequency of application during follow-up), and (3) investigate other relevant PROs assessed in the included studies (e.g. pain, depression, anxiety).

Methods: The search was conducted in MEDLINE, Embase, CINAHL, PsycINFO, CENTRAL, the trials registers ClinicalTrials.gov, and WHO ICTRP. All original empirical research published between January 2005 and April 2018 was included. Data extraction, narrative content analysis, and a critical appraisal were performed.

Results: The search yielded 3496 hits and 54 publications were included in the review. Predominantly, HRQoL was assessed (in 48 of 54 publications), with Short Form-36 Health Survey (SF-36) applied most frequently. ADL and (social) participation were rarely assessed. The follow-up period lasted between 8 days and 17.5 years; assessments were performed between one and five times. Most used were a single assessment of PROs, a follow-up period of 1 to 1.5 years, and a postal survey. Other relevant PRO areas reported were function, mental disorders, and pain.

Conclusions: There is a large variation in the assessment of PROs after polytrauma, impairing comparability of outcomes. First efforts to standardise the collection of PROs have been initiated, but further harmonisation between central players, such as trauma registers, is required. Definition and continuous collection of core outcome sets would improve comparability of study results, creating the basis for more targeted treatment. Additional knowledge on rarely reported PRO areas like (social) participation and social network may lead to their consideration in health services provision.

\section{Registration: PROSPERO (CRD42017060825)}

\section{Introduction}

Severe injuries represent a leading cause of death and permanent disability (1). In the central European region, severely injured people are referred to as having a 'polytrauma' or being 'polytraumatised' (2). A polytrauma is defined as at least two severe injuries in different body regions or organ systems, at least one of which is potentially life-threatening by itself or in combination with others, with an Injury Severity Score (ISS) $\geq 16$ (1-4). According to the TraumaRegister DGU®, a German registry that covers patients with severe injuries, the number of patients with polytrauma (ISS $\geq 16)$ was 15,651 in $2019(5)$. Most affected were men (72\%) with a mean age of 46.5 years (6).

The survival rates after polytrauma have increased lately, bringing the quality of survival into the focus of research. One year after polytrauma, survivors often fail to reach pre-traumatic status due to persisting medical problems (7). Disabilities such as respiratory problems, paraplegia or tetraplegia, prevent $40 \%$ from returning to their former workplace $(8,9)$. They also have an impact on socio-economic aspects and quality of life $(9-11)$. Therefore, it is crucial to reduce the short-term and long-term burden after polytrauma $(9,11)$. In this context, the patients' perspective on polytrauma outcomes focussing on psychological, physical and social factors is getting more important for the patients themselves, their relatives, and healthcare professionals (11, 12). Evaluating these patient-reported outcomes (PROs) is essential to complete the holistic assessment of relevant clinical outcomes after injury from a patient's perspective.

To assess the impact of polytrauma on health-related quality of life (HRQoL) and other important PROs, one must consult the person affected directly (12, 13). $\mathrm{HRQ}$ oL is already being recorded in registers like the Australian Victoria State Trauma Registry. The German TraumaRegister DGU ${ }$ will also complement its assessment with HRQoL (14). Activities of daily living (ADL), defined as basic activities to fulfil independent living, along with physical and psychological needs, are meaningful outcome parameters in case of post-polytrauma impairment. Participation is a health component according to the International Classification of Functioning, Disability and Health (ICF) (15). In recent years, it has gained more attention in political context (16). Several working groups have controversially discussed which tools should be applied to assess PROs after polytrauma, at which time points, and in which way (13, 17-25); however, guidelines with clear recommendations are lacking (13), and no systematic review could be identified.

Therefore, the aim of this systematic review was to:

1. collect instruments assessing HRQoL, ADL, and social participation during follow-up after polytrauma

2. describe their application in detail (e.g. duration of follow-up period, frequency of application, and time point of measurements within the followup period)

3. investigate which other relevant PROs are additionally assessed in included studies (e.g. pain, depression, anxiety, and cognitive function) without detailed analysis.

\section{Methods}

This systematic review was conducted in line with the Preferred Reporting Items for Systematic Review and Meta-Analysis (PRISMA) guideline (26) (Additional file 1). The review protocol was registered on 8 April 2017 at PROSPERO (CRD42017060825).

\section{Data Sources and Search Strategy}

The search was conducted in MEDLINE, Embase, CINAHL (Cumulative Index to Nursing and Allied Health Literature), PsycINFO, CENTRAL (Cochrane Central Register of Controlled Trials), the trials registers ClinicalTrials.gov and WHO ICTRP (WHO International Clinical Trials Registry Platform), covering the period from 1 January 2005 until 5 April 2018. In addition, reference lists of eligible studies were examined. Textwords (tw) and MeSH-terms (mh) like 'polytrauma' 
(tw), 'multiple trauma' (mh), 'quality of life' (mh), 'activities of daily living' (tw), 'social participation' (mh) and 'pain' (mh) were used. The development of the search strategy was outlined in the study protocol (27) and is provided in Additional file 2.

\section{Inclusion and Exclusion Criteria}

The publications included primarily investigated PROs of patients aged $18-75$ years with an Injury Severity Score (ISS) $\geq 16$ and/or an Abbreviated Injury Scale (AIS) $\geq 3$ during a clearly defined follow-up period. All original empirical research published in English or German was included. Publications examining special types of injury or illness - e.g. low-energy injuries, single or mono injuries, burn injuries, war injuries, or cancer - were excluded. We also excluded some publication types (grey literature, books, abstracts, and editorials). Systematic reviews and meta-analysis were not included, too, but screened to identify further appropriate studies. More details about the inclusion and exclusion criteria were published in the study protocol (27).

\section{Selection of Publications and Studies}

Four teams with two reviewers each (MR-IG; KM-SK; MR-SOB; Al-AHF) screened the title and abstracts independently. To foster the process of eligibility decisions, the inclusion or exclusion of the first 50 publications was discussed by two reviewers (MR-IG). In a next step, two reviewers (MR-IG; KM-SK; MR-SOB, KM-SOB) independently screened the full texts of the remaining publications for final inclusion. A third reviewer (MR, SK, IG) solved disagreements regarding the inclusion of publications for full-text screening and their final inclusion in this review. We identified publications relating to the same study and/or population. For this purpose, extensive research was conducted, and authors of the corresponding publications were contacted. The section 'Characteristics of included publications' outlines how we approached publications dealing with different research questions of the same study, comprising subpopulations drawn from a superior study population, reporting subprojects of a study project, or presenting results of the same research question at different follow-up periods.

\section{Data Extraction and Analysis}

Data extraction was performed according to the requirements of Cochrane reviews (28). For the first and second aim, two researchers (MR-IG) used a piloted data extraction sheet independently comprising the following: first author, publication year and country, study design, aim of the study (objective), study population, treatment, applied instruments (incl. modifications), time of measurements, length of follow-up period, method of assessment (application), study results, and results of the critical appraisal. Regarding the third aim, the other reported PROs were extracted and categorised in a separate extraction sheet. Narrative content analysis was performed regarding the assessment of HRQol, ADL and social participation as well as further reported PROs.

\section{Critical Appraisal}

Teams with two reviewers each (MR, IG, SK, KM, SOB) independently assessed the methodological quality and the risk of bias in each study type using standardised checklists of the UK National Institute for Health and Care Excellence (NICE) $(29,30)$, Scottish Intercollegiate Guidelines Network (SIGN) (31), or the Mixed Method Appraisal Tool (MMAT) (32). Discrepancies were resolved by discussion between the reviewers or by a third reviewer.

\section{Patient and Public Involvement Statement}

Patients were not involved in the design and implementation of this systematic review.

\section{Results \\ Search Results}

In total, 3485 references were retrieved. After a title and abstract screening as well as a full-text screening, further 11 records were identified by backward citation tracking. Finally, 54 eligible publications were included for data extraction (Fig. 1).

\section{Characteristics of Included Publications}

The publications ( $n=54)$ most often came from Germany $(n=15,28 \%)$ and the Netherlands $(n=11,20 \%)$; rarely from Austria, Denmark, France, Japan, Poland, Spain and Sweden (each $n=1,2 \%$ ). One study has been carried out in Germany and Austria, another one in Europe, Asia, Australia, Africa, North- and South-America. The publications primarily reported monocentric studies $(n=44,81 \%)$ and predominately comprised prospective cohort studies $(n=26,48 \%)$ (Table 1).

The number of participants varied between 8 (33) and 7612 (34), with an average age of $24.6 \pm 12.1$ (35) to $53.9 \pm 18.8$ (36) years, and a proportion of men between $37.5 \%$ (33) and $84 \%$ (37). The mean ISS ranged between $3.9 \pm 1.7(38)$ and 56.8 points $(39,40)$ and the AIS - reported in 18 publications - between 0 and 5 points. Information regarding treatment of injuries was provided in $n=31$ publications (Table 1 ).

Six publications were related to the Hannover Rehab study $(35,41-45)$. Four reported the results of a polytrauma study project conducted at the University Hospital Basel, which included a subproject of a study project (EK 159/02) and a polytrauma cohort (46-49). Study projects at the University Medical Centre Utrecht displayed almost identical recruitment periods and locations (Holtslag et al. 2007 a, b: January 1999 to December 2000 (50, 51), Holtslag et al. 2006 and 2008: January 1999 to January 2001 (52,53), Holtslag et al. (2006) analysed a subgroup out of $n=335$ participants (52); Holtslag et al. 2008 additionally included a group 'Fatalities' $(53)$. Two publications $(54,55)$ used the same data collection but addressed different research questions $(C I H R$, grants no. MOP62873 , FRSQ, grant no. 3255). The publication by Sirois et al. (2009) is the original study (55), the publication by Ouellet et al. (2009) is a secondary analysis (54). Three publications comprised patients treated at the Murnau Trauma Centre from 2000 to 2005 and recorded in the TraumaRegister DGUß (39, 40, 56). The publication by von Rueden et al. (2013) (39) and Wurm et al. (2012) (40) reported on the same investigation of a group of severely injured patients with an 
ISS > 50; Simmel et al. (2013) analysed those patients with an ISS > 25 (56). Soberg et al. (2011) (57) investigated the same research question as Soberg et al. (2007) (58) with a follow-up of 5 years (Table 1).

\section{Instruments Used to Assess HRQoL}

In total, 15 different tools were used to assess HRQoL during follow-up after polytrauma. Of those, six were generic instruments: Short Form-36 Health Survey $(S F-36, n=21,39 \%)(34,37,46-49,52,57-70), 12$-Item Short Form Health Survey (SF-12, $n=12,22 \%)(35,36,41-45,54,55,71-73)$, Nottingham Health Profile (NHP, $n=4,7 \%)(46-49)$, Sickness Impact Profile (SIP, $n=4,7 \%)(11,50,52,67)$, Short Musculoskeletal Function Assessment (SMFA, $n=1,2 \%)(74)$, and World Health Organization Quality of Life Instrument - Short Form (WHOQOL-BREF, $n=1,2 \%)(74)$. In addition, four preference-based tools were applied: European Quality of Life 5-Dimension (EQ-5D, $n=9,17 \%)(7,46-49,51,53,70,75)$, EQ-6D $(n=1,2 \%)(33)$, Health Utility Index Mark 2 (HUI 2, $n=1,2 \%)(75)$, and HUI $3(n=1,2 \%)(75)$. Three trauma-specific instruments were used: Hannover Score for Polytrauma Outcome (HASPOC, $n=6,11 \%)(35,41-45)$, Trauma Outcome Profile (TOP, $(n=4,7 \%)(46,48,49,70)$, and Aachen Long-term Outcome Score (ALOS, $n=1,2 \%)(46)$. The Polytrauma Outcome Chart (POLO Chart, $n$ $=6,11 \%)(38-40,56,76,77)$ was used as a modular instrument consisting of the Glasgow Outcome Profile, SF-36, EQ-5D, and TOP. The International Classification of Impairments, Disabilities and Handicaps (ICIDH) was reported in one publication $(n=1,2 \%)(78)$. Fifteen publications combined two to five different instruments to assess HRQoL $(35,41-49,52,67,70,74,75)$.

In some publications $(n=5)$, instruments to assess HRQoL were only used partially (e.g. solely the mental or physical part, or selected subscales of the SF-36 or SF-12, chapter 'Disability' of the ICIDH) $(34,54,55,71,78)$. Six publications conducted a retrospective collection of HRQoL via SF-36, SF-12, EQ-5D and TOP capturing the pre-trauma status $(37,46-49,73)$.

\section{Instruments Used to Assess ADL}

ADL was measured by different generic instruments: Groningen Activity Restriction Scale (GARS) (52), Functional Activities Questionnaire (FAQ) (63), Katz Index of Activities of Daily Living (KATZ ADL) (63), and Barthel Index (BI) (each $n=1,2 \%)(38)$. The SF-36 subscales of physical and social functioning ( $\mathrm{n}=1$, $2 \%)(79)$ were also used to record ADL. Two publications employed self-designed questions $(7,34)$. Most frequently $(n=5)$, a single instrument was applied to assess $\operatorname{ADL}(7,34,38,52,79)$.

\section{Instruments Used to Assess (Social) Participation}

One publication reported the assessment of participation by self-designed questions covering return to sports and work (52).

Table 2 displays the number of publications and the instruments used to assess HRQoL, ADL, and (social) participation. Application of Instruments

A postal survey was most often used to assess HRQoL, ADL, and (social) participation ( $n=17)(11,33,46-52,56,57,67,71,74,75,77,79)$. Next in frequency were telephone interviews $(n=5)(34,36,54,55,80)$, expert assessments $(n=2)(44,63)$, face to face interview $(n=1)(81)$, and combinations of those $(n=16)$ $(7,35,37,39,40,42,43,45,58,59,65,68,70,73,82,83)$. The research settings were: trauma centre $(35,42,43,45,84)$, outpatient clinic $(61,64,78)$, hospital (38), medical centre or place of patient's residence (63), hospital and home (73). In four publications, no information regarding the application of instruments was provided $(41,53,66,72)$. Relatives or proxies were reported in five publications $(36,51,52,55,62)$. While most instruments are used with several data collection methods and survey settings, the HASPOC, where reported, is only recorded in the trauma centre and is assessed by a physician in addition to the respondent.

\section{Measurements During Follow-Up}

PROs mentioned above were most frequently recorded at a single time point $(n=47)(7,11,33-35,38-46,48-56,59-63,65-72,74-84)$. Few publications reported two $(47,64,73)(n=3)$, three $(36,58)(n=2)$, four or five $(37,57)$ (each $n=1)$ time points of assessment.

\section{Duration of Follow-Up}

Most often $(n=13)$, a follow-up of 1 to 1.5 years was reported $(34,37,50-53,59,61,64,67,72,75,81)$. Six publications described a follow-up period of over 10 and up to 17.5 years $(35,41-45)$. Rarely, shorter follow-up periods of 8 days $(84)$ to six months $(73,79)$ were detected.

Table 1 provides an overview of the included publications.

\section{Further PROs Assessed}

Beyond HRQoL, ADL, and (social) participation, assessments of further PROs were reported in the included publications. Most frequently these comprised function, pain, and mental disorders (including depression and/or depressive symptoms, anxiety, and PTSD). Only few PROs focused on social support, cognition, subjective health status, and social network.

Table 3 provides an overview of the kind and number of further recorded PROs.

\section{Critical Appraisal Results}

Some methodological weaknesses were identified regarding the selection of the study population, consideration of confounders, data analysis and presentation of results, internal validity and generalisability of study results $(33,39-42,44,60,64,66,70,76-78,80,82,83)$. While most criteria of the critical appraisal were fulfilled, a moderate risk of bias was observed $(7,11,35-38,43,45-52,55,56,58,59,61,62,65,68,71-74,79,81,84)$. A low risk of bias was observed in eight publications $(34,53,54,57,63,67,69,75)$. The results of the critical appraisal are provided in Table 1. 


\section{Discussion}

\section{Main Findings}

The present systematic review identified 54 publications contributing to the research questions. A great variety was observed among all investigated aspects. HRQoL was the most frequently recorded PRO, and SF-36, SF-12 and EQ-5D were the most frequently applied instruments to assess it. Data on ADL and (social) participation were rarely reported. Most questionnaires were sent out per mail. Measurements were predominantly performed at a single time point, and the follow-up period lasted mainly between 1 and 1.5 years. Function, mental disorders and pain were the most frequently assessed categories of the other PROs.

\section{Instruments for the Assessment of HRQoL, ADL, and (Social) Participation}

In 48 of the included publications, HRQoL was assessed - predominantly with generic and preference-based HRQoL instruments such as SF-36, SF-12, EQ-5D, and NHP. Thus, over a long period of time, results are available for the impact of polytrauma on HRQoL and its possible change over time.

Unsurprisingly, the SF-36, one of the oldest and most widely used instrument for assessing HRQoL (85), is the most frequently reported instrument in publications included in this review. Along with the SF-12, SF tools accounted for $61 \%$ of all identified instruments. This result is supported by observations of other reviews conducted in the field of polytrauma (85-88).

In 1999, an international consensus conference on evidence-based guidelines for the systematic evaluation and assessment of HRQoL after polytrauma recommended the use of modular instruments comprising generic and trauma-specific aspects (89). Generic instruments have been applied continuously over a long time period, have been translated into different languages, and can be applied regardless of the underlying disease. They are suitable for assessing HRQoL after polytrauma since it can affect different body regions, show different injury patterns, and lead to a variety of complications. In contrast, traumaspecific instruments capture targeted relevant problems and measure the longitudinal change of HRQoL (90). Despite the recommendation, the POLO Chart is the only modular instrument comprising generic and trauma-specific aspects reported in the included publications (90). HASPOC, TOP and ALOS were the trauma-specific tools used. HASPOC is an instrument designed to evaluate the quality of rehabilitation. Since it comprises instruments assessing inter alia HRQoL, ADL, and function $(91,92)$, we decided to subsume it into the category 'HRQoL'.

Apart from assessing HRQoL, we were particularly interested in the measurement of ADL and social participation. Regarding the assessment of ADL, five different instruments and self-designed questions in six publications were observed in our review. (Social) participation was only reported once by Holtslag et al. (2006) (52). In summary, it was notable that ADL and (social) participation after polytrauma were seldom assessed. One reason might be that measures of $\mathrm{HRQ}$ oL or function already include several questions related to ADL and (social) participation. Predominantly, (social) participation is researched in people with brain or spinal cord injuries. A general understanding and clear definition of the participatory construct is still missing, which might be another reason for the low reporting rates of (social) participation. It has been argued that (social) participation should be one of the main aspects in rehabilitation after polytrauma (93), besides survival and function, and there are a number of instruments available to assess this PRO (94-96). This could also be assumed for ADL.

\section{Application of Instruments}

Most frequently, a postal questionnaire was used. Compared to personal interviews, questionnaires sent by mail are quicker and less expensive. In addition, potential bias introduced by an interviewer is excluded. However, it is not possible to verify the person answering the questionnaire, comprehension questions cannot be clarified, the full completion of the questionnaire cannot be guaranteed, and the proportion of non-responders is higher (97). The consultation of a proxy answering the questions was rarely reported. Injuries, e.g. traumatic brain injuries (TBI), may require the use of a proxy and could enable the collection of outcome data after polytrauma, despite a risk of bias $(97,98)$. The present systematic review excluded publications reporting single or mono injuries like single TBIs, which might be the reason only few publications including proxies were detected.

The retrospective assessment of the pre-trauma situation helps enable a comparison of results and obtain a holistic view of the outcome after an injury. Gross et al. $(2010,2012)$ as well as Gross and Amsler (2011) measured pre-fracture level of HRQoL over 2 years after trauma (47-49). However, there are limitations to this approach: Memories of the pre-traumatic situation can be distorted by various circumstances, which might increase the risk of recall bias ( 99 ). Large population screenings or the use of population norms might help to evaluate the pre-traumatic state of HRQoL $(19,46)$. Baseline assessments should ideally be made in the first week after the event. However, this is not always possible due to the severity of the injury. Another limitation might be that some instruments are not suitable for use in hospital. For example, changed behaviour patterns measured by the SIP can also result from the very fact of being an inpatient. Apart from HRQoL, functional recovery is also of great interest and an important factor for evaluating outcomes after a polytrauma. In comparison to HRQoL and due to better quantification, the retrospective evaluation of function is more feasible (19).

Most of the included publications reported a single assessment of the PROs. However, the course of recovery cannot be derived from a single measurement. The aforementioned consensus conference recommended measurements at three, twelve and 24 months after the event (18). Other groups suggested assessments of PROs after one, two, four and twelve months (100) or six and twelve months after the injury, and beyond twelve months in case of longer rehabilitation needs (13).

The most frequently reported follow-up period were 1 to 1.5 years. Six publications conducted follow-up periods of more than ten years.

\section{Further PROs Assessed}

Further reported PROs assessed mainly function, mental disorders, and pain - outcomes that are highly affected by polytrauma. Social support, subjective health status, cognition, or social network were rarely recorded. This might be explained as follows: Instruments assessing HRQoL mainly capture subjective

Page 5/13 
health status. While cognition is frequently affected by brain injuries, publications reporting study populations with single brain injuries were excluded from this review. Social network and social support seem to influence recovery and return to work after trauma $(101,102)$ and should therefore receive more attention in future research.

\section{Recommendations for the Assessment of PROs}

The existing recommendations for the use and application of instruments - as well as time points of assessments and outcomes to be measured after polytrauma - differ substantially $(13,18)$, but there are attempts to standardise outcome measurements $(19)$. Classification systems might be helpful for a standardised assessment of PROs, facilitating the adequate selection of instruments for a specific research objective (24). The internet platform PROMIS Patient-reported Outcomes Measurement Information System - provides research tools and is continuously updated with the latest developments and newly translated instruments (103). An important step towards a standardised approach of collecting HRQoL was made in the TraumaRegister DGUß by the implementation of HRQoL assessment with the SF-12 (14).

Furthermore, several groups have focused on the definition of special outcome variables to be recorded as default parameters. Hoffman et al. (2016) recommended the development of a 'minimum data set' based on the ICF (21). A core outcome set relevant for the respective outcome could be defined. This procedure could guide decision-making for the collection and presentation of outcomes in studies (23). In addition, ICF linking enabling a check of which ICF components are mapped by an instrument could support the tool selection for particular research questions $(17,20)$. Other authors also support the idea of core outcome sets $(22,25)$.

\section{Strengths and Limitations}

The present systematic review has some limitations: Selection bias can be assumed as the search was restricted by the search period and language. However, we performed backward citation tracking and saw that only a few additional articles could be included.

This review was conducted thoroughly based on a registered protocol in line with the PRISMA-P quality and the PRISMA statement requirements. We provide a broad overview of instruments used for the collection of HRQoL, ADL and (social) participation, along with information on their application. In addition, this study offers an overview on further PROs reported in the context of polytrauma.

\section{Conclusion}

The present review reveals variations across study findings, which impairs the comparability of outcomes after polytrauma. To improve this situation, a more standardised approach for recording PROs should be established. First efforts have been initiated, but further alignment between the central players, such as the trauma registers, is required. Continuous collection of core outcomes during the regeneration of persons with polytrauma could create the basis for a targeted use of treatments and their adaptation to the results of the outcome measurement. Moreover, there appears to be little research on (social) participation and on further PROs like social support and social network. PROs need more attention in polytrauma-related outcome research. After all, we need to know the patients' perspectives on relevant outcomes after polytrauma so as to consider their needs when providing health services.

\section{Abbreviations}

ADL: Activities of daily living; AH-F: Annegret Herrmann-Frank; Al: Andrea Icks; AIS: Abbreviated Injury Scale; AIS $\S$ : AIS values are shown in publication for various body regions; ALOS: Aachen Long-term Outcome Score; AUC: Academy for Trauma Surgery GmbH; BI: Barthel Index; cs: can't say; ct: can't tell; CENTRAL: Cochrane Central Register of Controlled Trials; CINAHL: Cumulative Index to Nursing and Allied Health Literature; CW: Charlotte Wittgens; DALY: Disability-adjusted life year; e.g.: For example; EQ-5D: European Quality of Life 5-Dimension; EQ-6D: Six-Dimensional EuroQol instrument; EQ-VAS: EQ-5D visual analogue scale; F: Female; FAQ: Functional Activities Questionnaire; Fig.: Figure; FT: Fast track; GARS: Groningen Activity Restriction Scale; HASPOC: Hannover Score for Polytrauma Outcome; HRQoL: Health-related quality of life, HUI 2: Health Utility Index Mark 2; HUI 3: Health Utility Index Mark 3; ICF: International Classification of Functioning, Disability and Health; ICIDH: International Classification of Impairments, Disabilities and Handicaps; ICU: Intensive Care Unit; IDI: injury distress index; IG: Irmela Gnass; IQR/IR: Interquartile range; ISS: Injury Severity Score; JS: Johannes Sturm; JW: Joachim Windolf; KATZ ADL: Katz Index of Activities of Daily Living; KM: Kai Moschinski; M: Male; max.: highest value; MCS: Mental Component Summary score; Md: Median; MeSH: Medical subject heading; mh: MeSH-term; MIM: Maria-Inti Metzendorf; MMAT: Mixed Method Appraisal Tool; MR: Michaela Ritschel; MULT: Patients after polytrauma; N: Number; n: no; NA: not applicable; NHP: Nottingham Health Profile; NICE: UK National Institute for Health and Care Excellence; NR: not reported; NRTW: Nonreturn to work; N-WCP: Non-workers' compensation patients; PCS: Physical Component Summary score; POLO Chart: Polytrauma-Outcome-Chart; PRISMA: Preferred Reporting Items for Systematic Review and Meta-Analysis; PRISMA-P: Preferred Reporting Items for Systematic Review and Meta-Analysis Protocols; PRO: Patient-reported outcome; PROMIS: Patient-reported Outcomes Measurement Information System; PTSD: Posttraumatic stress disorder; PTSS: Posttraumatic stress symptoms; QoL: Quality of Life; SA: Silke Andrich; SBI: Severe brain injuries; SK: Silke Kuske; RTW: Return to work; SD: Standard deviation; SF: Sascha Flohé; SF-12: 12-Item Short Form Health Survey; SF-36: Short Form-36 Health Survey; SIGN: Scottish Intercollegiate Guidelines Network; SIP: Sickness Impact Profile; SMFA: Short Musculoskeletal Function Assessment; SOB: Sandra Olivia Borgmann; TBI: traumatic brain injury; TOP: Trauma Outcome Profile; tw: Textwords; WCP: Workers' compensation patients; WHO ICTRP: WHO International Clinical Trials Registry Platform WHOQOL-BREF: World Health Organization Quality of Life Instrument - Short Form; y: yes; YLD: Years lived with disability; YLL: Years of life lost

\section{Declarations}

\section{Acknowledgments}

Not applicable. 


\section{Authors' contributions}

Al is the guarantor. MIM, MR, SK, IG, SA, and AH-F developed the search strategy. MR, SK, IG, SA, KM, AH-F, MIM, SF, JS, JW, and Al contributed to the development of the selection criteria, the risk of bias assessment strategy, and data extraction criteria. MR, SK, IG, KM, SOB, AH-F, and Al conducted the screenings. The critical appraisal was performed by MR, SK, IG, KM, and SOB. Data extraction was carried out by MR, IG, and CW. MR, SK, IG, SA, and AI drafted the manuscript. MR, SK, IG, SA, KM, SOB, AH-F, MIM, CW, SF, JS, JW, and Al read the manuscript, provided feedback, and approved the final version.

\section{Competing interests}

JS was executive secretary of the Academy for Trauma Surgery GmbH (AUC). The authors declare that they have no competing interests.

\section{Funding}

This systematic review was supported by the Academy for Trauma Surgery GmbH (AUC). The funding supported the conduct of this systematic review.

\section{Availability of data and materials}

Not applicable.

\section{Consent for publication}

Not applicable.

\section{Ethics approval and consent to participate}

Not applicable.

\section{Differences between protocol and review}

We have planned to include publications only when the full text of the study is available. However, since all texts were available, there was nothing to exclude according to this criterion.

\section{References}

1. Ringburg AN, Polinder S, van lerland MC, Steyerberg EW, van Lieshout EM, Patka P, et al. Prevalence and prognostic factors of disability after major trauma. J Trauma. 2011;70(4):916-22.

2. Paffrath T, Lefering R, Flohe S. How to define severely injured patients? - An Injury Severity Score (ISS) based approach alone is not sufficient. Injury. 2014;45 Suppl 3:S64-9.

3. Mörsdorf P, Becker S, Holstein J, Burkhardt M, Pohlemann T. [Lebensqualität nach Polytrauma]. Chirurg. 2014;85:208-14.

4. Deutsche Gesellschaft für Unfallchirurgie e. V. [Weißbuch Schwerverletztenversorgung]. https://www.dgu-online.de/q-s/schwerverletzte/weissbuchschwerverletztenversorgung.html. Accessed 10 Nov 2019.

5. Sektion Notfall- \& Intensivmedizin \& Schwerverletztenversorgung der Deutschen Gesellschaft für Unfallchirurgie e.V. und AUC - Akademie der Unfallchirurgie GmbH. [TraumaRegister DGU® - Jahresbericht 2020]. http://www.traumaregister-dgu.de/fileadmin/user_upload/traumaregisterdgu.de/docs/Downloads/Jahresbericht_2020.pdf. Accessed 01 Oct 2020.

6. Pape-Köhler CIA, Simanski C, Nienaber U, Lefering R. External factors and the incidence of severe trauma: Time, date, season and moon. Injury. 2014;45 Supplement 3:S93-S9.

7. Vles WJ, Steyerberg EW, Essink-Bot ML, van Beeck EF, Meeuwis JD, Leenen LP. Prevalence and determinants of disabilities and return to work after major trauma. J Trauma. 2005;58:126-35.

8. Debus F, Lefering R, Frink M, Kuhne CA, Mand C, Bucking B, et al. [Numbers of Severely Injured Patients in Germany. A Retrospective Analysis From the DGU (German Society for Trauma Surgery) Trauma Registry.]. Dtsch Arztebl Int. 2015;112:823-9.

9. Hayes JA, Black NA, Jenkinson C, Young JD, Rowan KM, Daly K, et al. Outcome measures for adult critical care: a systematic review. Health Technol Assess. 2000;4:1-111.

10. Haeusler JM, Tobler B, Arnet B, Huesler J, Zimmermann H. Pilot study on the comprehensive economic costs of major trauma: consequential costs are well in excess of medical costs. J Trauma. 2006;61:723-31.

11. Post RB, van der Sluis CK, Ten Duis HJ. Return to work and quality of life in severely injured patients. Disabil Rehabil. 2006;28:1399-404.

12. Sittaro NA, Lohse R, Panzica M, Probst C, Pape HC, Krettek C. [Hannover-Polytrauma-Longterm-Study HPLS]. Versicherungsmedizin. 2007;59:20-5.

13. Ardolino A, Sleat G, Willett K. Outcome measurements in major trauma - results of a consensus meeting. Injury. 2012;43:1662-6.

Page $7 / 13$ 
14. Kamp O, Pfeifer R, Ritschel M, Flohe S, Bieler D. Polytrauma outcome: implementation of health-related quality of life assessment into the German Trauma Registry. Eur J Trauma Emerg Surg. 2019.

15. World Health Organization. International Classification of Functioning, Disability and Health: Children \& Youth Version: ICF-CY. Geneva: World Health Organization. http://apps.who.int/iris/bitstream/10665/43737/1/9789241547321_eng.pdf. Accessed 20 May 2017.

16. Farin E. ["Teilhabe" (Social Participation) of Patients in Health Care Research: Relations to Similar Concepts and Overview of Assessment Instrumets]. Gesundheitswesen. 2010;73:e1-e11.

17. Ballert CS, Hopfe M, Kus S, Mader L, Prodinger B. Using the refined ICF Linking Rules to compare the content of existing instruments and assessments: A systematic review and exemplary analysis of instruments measuring participation. Disabil Rehabil. 2016:1-17.

18. Bouillon B, Kreder H. Quality of life in patients with multiple injuries - basic issues, assessment, and recommendations. Restor Neurol Neurosci. 2002;20:125-34.

19. Cameron PA, Gabbe BJ, McNeil JJ. The importance of quality of survival as an outcome measure for an integrated trauma system. Injury. 2006;37:117884.

20. Cieza A, Fayed N, Bickenbach J, Prodinger B. Refinements of the ICF Linking Rules to strengthen their potential for establishing comparability of health information. Disabil Rehabil. 2019;41:574-83.

21. Hoffman KP, Playford DE, Grill E, Soberg HL, Brohi K. Minimum data set to measure rehabilitation needs and health outcome after major trauma: Application of an international framework. Eur J Phys Rehabil Med. 2016;52:331-46.

22. Prinsen CA, Vohra S, Rose MR, Boers M, Tugwell P, Clarke M, et al. How to select outcome measurement instruments for outcomes included in a "Core Outcome Set" - a practical guideline. Trials. 2016;17:449.

23. Selb M, Escorpizo R, Kostanjsek N, Stucki G, Üstün B, Cieza A. A guide on how to develop an International Classification of Functioning, Disability and Health Core Set. Eur J Phys Rehabil Med. 2015;51:105-17.

24. Valderas JM, Alonso J. Patient reported outcome measures: A model-based classification system for research and clinical practice. Qual Life Res. 2008;17:1125-35.

25. Williamson PR, Altman DG, Blazeby JM, Clarke M, Devane D, Gargon E, et al. Developing core outcome sets for clinical trials: Issues to consider. Trials. 2012;13:132.

26. Moher D, Liberati A, Tetzlaff J, Altman DG, PRISMA Group. Prefered reporting items for systematic reviews and meta-analyses: the PRISMA statement. PLoS Med. 2009;6:e1000097.

27. Gnass I, Ritschel M, Andrich S, Kuske S, Moschinski K, Herrmann-Frank A, et al. Assessment of patient-reported outcomes after polytrauma: protocol for a systematic review. BMJ Open. 2018;8(e017571).

28. The Cochrane Collaboration. Cochrane Handbook for Systematic Reviews of Interventions Version 5.1.0. 2011. http://handbook.cochrane.org. Accessed 03 Jan 2017.

29. National Institute for Health and Care Excellence (NICE). Nice Appendix F Quality appraisal checklist - quantitative intervention studies. https://www.nice.org.uk/process/pmg4/chapter/appendix-f-quality-appraisal-checklist-quantitative-intervention-studies\#notes-on-the-use-of-thequantitative-studies-checklist. Accessed 08 Dec 2016.

30. National Institute for Health and Care Excellence (NICE). NICE Appendix G Quality appraisal checklist - quantitative studies reporting correlations and associations. https://www.nice.org.uk/process/pmg4/chapter/appendix-g-quality-appraisal-checklist-quantitative-studies-reporting-correlations-and. Accessed 08 Dec 2016.

31. Scottish Intercollegiate Guidelines Network (SIGN). SIGN Methodology Checklist 5: Studies of Diagnostic Accuracy. http://www.google.de/url? $s a=t \& r c t=j \& q=\& e s r c=s \& s o u r c e=w e b \& c d=4 \& c a d=r j a \& u a c t=8 \& s q i=2 \& v e d=0$ ahUKEwiYp9P824bVAhWLJIAKHSmKDZcQFgg7MAM\&url=http\%3A\%2F\%2Fww studies.doc\&usg=AFQjCNETExuNUQoaEFhPoPXgk5uw-yXtGQ. Accessed 08 Dec 2016.

32. Pace R, Pluye P, Bartlett G, Macaulay AC, Salsberg J, Jagosh J, et al. Testing the reliability and efficiency of the pilot Mixed Methods Appraisal Tool (MMAT) for systematic mixed studies review. Int J Nurs Stud. 2012;49:47-53.

33. Gribnau AJG, van Hensbroek PB, Haverlag R, Ponsen KJ, Been HD, Goslings JC. U-shaped sacral fractures: Surgical treatment and quality of life. Injury. 2009;40:1040-8.

34. McCarthy ML, Dikmen SS, Langlois JA, Selassie AW, Gu JK, Horner MD. Self-reported psychosocial health among adults with traumatic brain injury. Arch Phys Med Rehabil. 2006;87:953-61.

35. Zelle BA, Panzica M, Vogt MT, Sittaro NA, Krettek C, Pape HC. Influence of workers' compensation eligibility upon functional recovery 10 to 28 years after polytrauma. Am J Surg. 2005b;190:30-6.

36. Marasco S, Lee G, Summerhayes R, Fitzgerald M, Bailey M. Quality of life after major trauma with multiple rib fractures. Injury. 2015;46:61-5.

37. Bouman A, Hemmen B, Evers S, van de Meent H, Ambergen T, Vos P, et al. Effects of an Integrated 'Fast Track' Rehabilitation Service for Multi-Trauma Patients: A Non-Randomized Clinical Trial in the Netherlands PLOS One. 2017;12:e0170047.

38. Lefering R, Tecic T, Schmidt Y, Pirente N, Bouillon B, Neugebauer E. Quality of life after multiple trauma: Validation and population norm of the Polytrauma Outcome (POLO) Chart. Eur J Trauma Emerg Surg. 2012;38:403-15.

39. von Rüden C, Woltmann A, Rose M, Wurm S, Ruger M, Hierholzer C, et al. Outcome after severe multiple trauma: A retrospective analysis. J Trauma Manag Outcomes. 2013;7:4.

40. Wurm S, Rose M, von Ruden C, Woltmann A, Buhren V. [Severe polytrauma with an ISS Z 50]. Z Orthop Unfall. 2012;150:296-301. 
41. Andruszkow H, Probst C, Grün O, Krettek C, Hildebrand F. Does additional head trauma affect the long-term outcome after upper extremity trauma in multiple traumatized patients: is there an additional effect of traumatic brain injury? Clin Orthop Relat Res. 2013;471:2899-905.

42. Dienstknecht T, Pfeifer R, Horst K, Sellei RM, Berner A, Zelle BA, et al. The long-term clinical outcome after pelvic ring injuries. Bone Joint J. 2013;95:54853.

43. Probst C, Zelle B, Panzica M, Lohse R, Sitarro NA, Krettek C, et al. Clinical re-examination 10 or more years after polytrauma: Is there a gender related difference? J Trauma. 2010;68:706-11.

44. Schmidt-Rohlfing B, Pfeifer R, Kaneshige J, Hofman M, Knobe M, Sellei R, et al. Scoring systems for outcome after knee injuries. Injury. 2011;42:271-5.

45. Zelle B, Brown S, Panzica M, Lohse R, Sittaro N, Krettek C, et al. The impact of injuries below the knee joint on the long-term functional outcome following polytrauma. Injury. 2005a;36:169-77.

46. Attenberger C, Amsler F, Gross T. Clinical evaluation of the Trauma Outcome Profile (TOP) in the longer-term follow-up of polytrauma patients. Injury. 2012;43:1566-74

47. Gross T, Attenberger C, Huegli RW, Amsler F. Factors associated with reduced longer-term capacity to work in patients after polytrauma: A Swiss trauma center experience. J Am Coll Surg. 2010;211:81-91.

48. Gross T, Amsler F. Prevalence and incidence of longer term pain in survivors of polytrauma. Surgery. 2011;150:985-95.

49. Gross T, Schuepp M, Attenberger C, Pargger H, Amsler F. Outcome in polytraumatized patients with and without brain injury. Acta Anaesthesiol Scand. 2012;56:1163-74.

50. Holtslag HR, Post MW, Lindeman E, Van der Werken C. Long-term functional health status of severely injured patients. Injury. 2007a;38:280-9.

51. Holtslag HR, van Beeck EF, Lindeman E, Leenen LP. Determinants of long-term functional consequences after major trauma. J Trauma. 2007b;62:919-27.

52. Holtslag H, Buskens E, Rommers C, Prevo A, van der Werken C. Long-term outcome after lower extremity injuries in severely injured patients. Euro J Trauma. 2006;32:365-73.

53. Holtslag HR, van Beeck EF, Lichtveld RA, Leenen LP, Lindeman E, van der Werkend C. Individual and population burdens of major trauma in the Netherlands. Bull World Health Organ. 2008;86:111-7.

54. Ouellet MC, Sirois MJ, Lavoie A. Perceived mental health and needs for mental health services following trauma with and without brain injury. J Rehabil Med. 2009;41:179-86.

55. Sirois MJ, Dionne CE, Lavoie A. Regional differences in rehabilitation needs, rehabilitation access, and physical outcomes among multiple trauma survivors. Am J Phys Med Rehabil. 2009;88:387-98.

56. Simmel S, Drisch S, Haag S, Buhren V. [Long-term results after multiple trauma with ISS > 25: Outcome and predictors of quality of life]. Chirurg. 2013;84:771-9.

57. Soberg HL, Roise O, Bautz-Holter E, Finset A. Returning to work after severe multiple injuries: Multidimensional functioning and the trajectory from injury to work at 5 years. J Trauma 2011;71:425-34.

58. Soberg HL, Finset A, Bautz-Holter E, Sandvik L, Roise O. Return to work after severe multiple injuries: A multidimensional approach on status 1 and 2 years postinjury. J Trauma 2007;62:471-81.

59. Abraham CM, Obremskey WT, Song Y, Jackson JC, Ely EW, Archer KR. Hospital delirium and psychological distress at 1 year and health-related quality of life after moderate-to-severe traumatic injury without intracranial hemorrhage. Arch Phys Med Rehabil. 2014;95:2382-9.

60. Ahrberg AB, Leimcke B, Tiemann AH, Josten C, Fakler JK. Missed foot fractures in polytrauma patients: a retrospective cohort study. Patient safety in surgery. 2014;8:10.

61. Baranyi A, Leithgob O, Kreiner B, Tanzer K, Ehrlich G, Hofer HP, et al. Relationship between posttraumatic stress disorder, quality of life, social support, and affective and dissociative status in severely injured accident victims 12 months after trauma. Psychosomatics. 2010;51:237-47.

62. Burghofer K, Lackner CK, Stolpe E, Schlechtriemen T, Mutschler WE. [Lebensqualität 5 Jahre nach schwerem stumpfem Trauma]. Notfall + Rettungsmedizin. 2005;8:552-63.

63. Jackson JC, Obremskey W, Bauer R, Greevy R, Cotton BA, Anderson V, et al. Long-term cognitive, emotional, and functional outcomes in trauma intensive care unit survivors without intracranial hemorrhage. J Trauma. 2007;62:80-8.

64. Lippert-Grüner M, Maegele M, Haverkamp H, Klug N, Wedekind C. Health-related quality of life during the first year after severe brain trauma with and without polytrauma. Brain Injury. 2007;21:451-5.

65. Overgaard M, Hoyer CB, Christensen EF. Long-term survival and health-related quality of life 6 to 9 years after trauma. J Trauma. 2011;71:435-41.

66. Renovell-Ferrer P, Bertó-Martí X, Diranzo-García J, Barrera-Puigdorells L, Estrems-Díaz V, Silvestre-Muñoz A, et al. Functional outcome after calcaneus fractures: a comparison between polytrauma patients and isolated fractures. Injury. 2017:S91-S6.

67. Sampalis JS, Liberman M, Davis L, Angelopoulos J, Longo N, Joch M, et al. Functional status and quality of life in survivors of injury treated at tertiary trauma centers: What are we neglecting? J Trauma. 2006;60:806-13.

68. Sluys K, Haggmark T, Iselius L. Outcome and quality of life 5 years after major trauma. J Trauma. 2005;59:223-32.

69. Suzuki T, Shindo M, Soma K, Minehara H, Nakamura K, Uchino M, et al. Long-term functional outcome after unstable pelvic ring fracture. J Trauma. 2007;63:884-8.

70. Zwingmann J, Hagelschuer P, Langenmair E, Bode G, Herget G, Sudkamp NP, et al. Lower health-related quality of life in Polytrauma Patients: Long-term follow-up after over 5 years. Medicine (Baltimore). 2016;95:e3515.

Page 9/13 
71. Archer KR, Abraham CM, Song Y, Obremskey WT. Cognitive-behavioral determinants of pain and disability two years after traumatic injury: A crosssectional survey study. J Trauma. 2012;72:473-9.

72. Tee JW, Chan CHP, Gruen RL, Fitzgerald MCB, Liew SM, Cameron PA, et al. Early predictors of health-related quality of life outcomes in polytrauma patients with spine injuries: A level 1 trauma center study. Global Spine J. 2014;4:21-32.

73. Williamson OD, Gabbe BJ, Cameron P, Edwards ER, Richardson MD. Predictors of moderate or severe pain 6 months after orthopaedic injury: a prospective cohort study. J Orthop Trauma. 2009;23:139-44.

74. van Delft-Schreurs C, van Son M, de Jongh M, Gosens T, Verhofstad M, de Vries J. Psychometric properties of the Dutch Short Musculoskeletal Function Assessment (SMFA) questionnaire in severly injured patients. Injury. 2016;47:2034-40.

75. Ringburg AN, Polinder S, Van lerland MCP, Steyerberg EW, Van Lieshout EMM, Patka P, et al. Prevalence and prognostic factors of disability after major trauma. J Trauma. 2011;70:916-22.

76. Christensen MC, Banner C, Lefering R, Vallejo-Torres L, Morris S. Quality of life after severe trauma: Results from the global trauma trial with recombinant factor VII. J Trauma. 2011;70:1524-31.

77. Kaske S, Lefering R, Trentzsch H, Driessen A, Bouillon B, Maegele M, et al. Quality of life two years after severe trauma: A single centre evaluation. Injury. 2014;45:S100-S5.

78. Hladki W, Lorkowski J, Trybus M, Brongel L, Kotela I, Golec E. Quality of life as a result of multiple injury in the aspect of limitations of handicaps-social roles. Przeglad Lekarski. 2009;66:134-40.

79. Stulemeijer M, van der Werf S, Jacobs B, Biert J, van Vugt AB, Brauer JM, et al. Impact of additional extracranial injuries on outcome after mild traumatic brain injury. J Neurotrauma. 2006;23:1561-9.

80. Livingston DH, Tripp T, Biggs C, Lavery RF. A fate worse than death? Long-term outcome of trauma patients admitted to the surgical intensive care unit. J Trauma. 2009;67:341-8.

81. Frénisy MC, Bénony $H$, Chahraoui $K$, Minot D, d'Athis P, Pinoit JM, et al. Brain injured patients versus multiple trauma patients: Some neurobehavioral and psychopathological aspects. J Trauma. 2006;60:1018-26.

82. Quale AJ, Schanke AK, Froslie KF, Roise 0 . Severity of injury does not have any impact on posttraumatic stress symptoms in severely injured patients. Injury. 2009;40:498-505.

83. Richter JC, Waydhas C, Pajonk FG. Incidence of posttraumatic stress disorder after prolonged surgical intensive care unit treatment. Psychosomatics. 2006;47:223-30.

84. Victorson DE, Enders CK, Burnett KF, Ouellette EA. The Injury Distress Index: development and validation. Arch Phys Med Rehabil. 2008;89:1893-902.

85. Hoffman K, Cole E, Playford ED, Grill E, Soberg HL, Brohi K. Health outcome after major trauma: what are we measuring? PLoS One. $2014 ; 9$ :e103082.

86. Grill E, Mittrach R, Müller M, Mutschler W, Schwarzkopf SR. [Outcomes der Funktionsfähigkeit bei Polytrauma]. Unfallchirurg. 2010;113:448-55.

87. Khan F, Amatya B, Hoffman K. Systematic review of multidisciplinary rehabilitation in patients with multiple trauma. Br J Surg. 2012;99 Suppl 1:88-96.

88. Hofhuis JGM, Spronk PE. Health-related quality of life and influence of age after trauma: An overview. J Trauma Acute Care Surg. 2014;76:549-56.

89. Neugebauer E, Bouillon B, Bullinger M, Wood-Dauphinee S. Quality of life after multiple trauma-summary and recommendations of the consensus conference. Restor Neurol Neurosci. 2002;20:161-7.

90. Pirente N, Bouillon B, Schäfer B, Raum M, Helling H, Berger E, et al. A systematically developed instrument for the assessment of health-related quality of life in multiple injured patients. The Polytrauma Outcome (POLO) Chart. Unfallchirurg. 2002;105:413-22.

91. Stalp M, Koch C, Regel G, Krettek C, Pape HC. [Development of a standardized instrument for quantitative and reproducible rehabilitation data assessment after polytrauma (HASPOC)]. Chirurg. 2001;72:312-8.

92. Stalp M, Koch C, Ruchholtz S, Regel G, Panzica M, Krettek C, et al. Standardized outcome evaluation after blunt multiple injuries by scoring systems: a clinical follow-up investigation 2 years after injury. J Trauma. 2002;52:1160-8.

93. Simmel S, Bühren V. [Polytrauma überlebt - und was kommt dann]? Unfallchirurg. 2009;112:965-74.

94. Resnik L, Plow M. Measuring Participation as Defined by the International Classification of Functioning, Disability and Health: An Evaluation of Existing Measures. Arch Phys Med Rehabil. 2009;90:856-66.

95. Magasi S, Post MW. A Comparative Review of Contemporary Participation Measures' Psychometric Properties and Content Coverage. Arch Phys Med Rehabil. 2010;91:S17-S28.

96. Eyssen I, Steultjens MP, Dekker J, Terwee CB. A Systematic Review of Instruments Assessing Participation: Challenges in Defining Participation. Arch Phys Med Rehabil. 2011;92:983-97.

97. Pfaff H, Bentz J, Ommen O, Ernstmann N. [Qualitative und quantitative Methoden der Datengewinnung]. In: Schwartz FW, Walter U, Siegrist J, Kolip P, Leidl R, Dierks ML, et al., editors. Public Health - Gesundheit und Gesundheitswesen. 3. Auflage. München: Urban \& Fischer; 2012 . p. $451-67$.

98. Bitzer EM. [Die Perspektive der Patienten - Lebensqualität und Patientenzufriedenheit]. In: Schwartz FW, Walter U, Siegrist J, Kolip P, Leidl R, Dierks ML, et al., editors. Public Health Gesundheit und Gesundheitswesen. 3rd edition. München: Urban \& Fischer; 2012. p. 485-91.

99. Scholten AC, Haagsma JA, Steyerberg EW, van Beeck EF, Polinder S. Assessment of pre-injury health-related quality of life: A systematic review. Popul Health Metr. 2017;15:10.

100. Van Beeck EF, Larsen CF, Lyons RA, Meerding WJ, Mulder S, Essink-Bot ML. Guidelines for the conduction of follow-up studies measuring injury-related disability. J Trauma. 2007;62:534-50. 
101. Prang KH, Berecki-Gisolf J, Newman S. Recovery from musculoskeletal injury: the role of social support following a transport accident. Health and Quality of Life Outcomes. 2015;13:97.

102. De Graaf MW, Reinings IHF, Heineman E, El Moumni M. The development and internal validation of a model to predict functional recovery after trauma. PLoS One. 2019;14:e0213510.

103. PROMIS Germany. Dynamic Tools to Measure Health Outcomes from the Patient Perspective. http://promis-germany.de. Accessed 15 June 2017.

\section{Tables}

Due to technical limitations, table 1 docx is only available as a download in the Supplemental Files section.

Table 2

Quantitative overview of instruments assessing HRQoL, ADL and (social) participation in $\mathrm{n}=54$ publications

\begin{tabular}{|c|c|c|c|}
\hline PRO & Category & Instrument & $N(\%)$ \\
\hline \multirow[t]{15}{*}{ HRQoL } & \multirow[t]{6}{*}{ Generic instruments } & Short Form-36 Health Survey (SF-36) & $21(39)$ \\
\hline & & 12-Item Short Form Health Survey (SF-12) & $12(22)$ \\
\hline & & Nottingham Health Profile (NHP) & $4(7)$ \\
\hline & & Sickness Impact Profile (SIP) & $4(7)$ \\
\hline & & Short Musculoskeletal Function Assessment (SMFA) & $1(2)$ \\
\hline & & $\begin{array}{l}\text { World Health Organization Quality of Life Instrument - Short Form (WHOQOL- } \\
\text { BREF) }\end{array}$ & $1(2)$ \\
\hline & \multirow{4}{*}{$\begin{array}{l}\text { Preference-based } \\
\text { instruments }\end{array}$} & European Quality of Life 5-Dimension (EQ-5D) & $9(17)$ \\
\hline & & EQ-6D* & $1(2)$ \\
\hline & & Health Utility Index Mark 2 (HUI 2) & $1(2)$ \\
\hline & & Health Utility Index Mark 3 (HUI 3) & $1(2)$ \\
\hline & \multirow[t]{3}{*}{ Trauma-specific instruments } & Hannover Score for Polytrauma Outcome (HASPOC) & $6(11)$ \\
\hline & & Trauma Outcome Profile (TOP) & $4(7)$ \\
\hline & & Aachen Long-term Outcome Score (ALOS) & $1(2)$ \\
\hline & Modular instruments & Polytrauma-Outcome-Chart (POLO Chart) & $6(11)$ \\
\hline & Conceptual framework & $\begin{array}{l}\text { The International Classification of Impairments, Disabilities and Handicaps } \\
\text { (ICIDH) }\end{array}$ & $1(2)$ \\
\hline \multirow[t]{6}{*}{$A D L$} & & Self-designed questions & $2(4)$ \\
\hline & \multirow[t]{5}{*}{ Generic instruments } & Groningen Activity Restriction Scale (GARS) & \multirow{5}{*}{$\begin{array}{l}\text { each } n=1 \\
(2)\end{array}$} \\
\hline & & Functional Activities Questionnaire (FAQ) & \\
\hline & & Katz Index of Activities of Daily Living (KATZ ADL) & \\
\hline & & Barthel Index (BI) & \\
\hline & & SF-36 subscales physical and social functioning & \\
\hline $\begin{array}{l}\text { (Social) } \\
\text { Participation }\end{array}$ & & Self-designed questions & $1(2)$ \\
\hline
\end{tabular}


Table 3

Quantitative overview of kind and number of further reported PROs in $n=54$

\begin{tabular}{|ll|}
\multicolumn{2}{|c}{ publications } \\
\hline PRO & N (\%) \\
\hline Function & $19(35)$ \\
\hline Mental disorder & $16(30)$ \\
\hline Pain & $14(26)$ \\
\hline Social support & $7(13)$ \\
\hline Cognition & $6(11)$ \\
\hline Subjective health status & $3(5)$ \\
\hline Social network & $2(4)$ \\
\hline Neuropsychological disorder & $1(2)$ \\
\hline
\end{tabular}

\section{Figures}

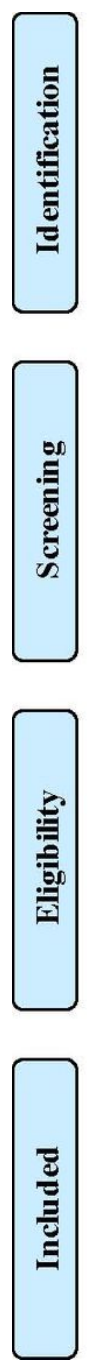

\section{Records identified through database} searching $(\mathrm{n}=3485)$
Additional records identified by backward citation tracking $(\mathrm{n}=11)$

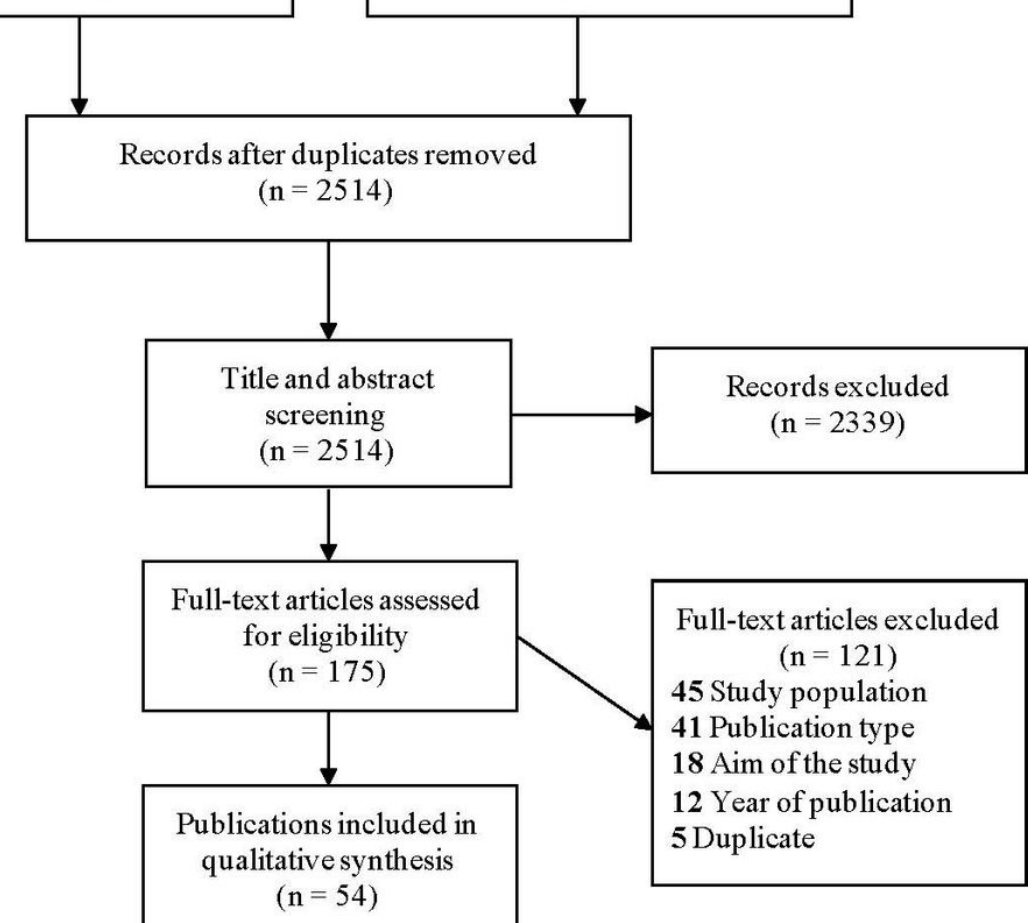

Figure 1

Flow chart

\section{Supplementary Files}

This is a list of supplementary files associated with this preprint. Click to download. 
- Table1.docx

- Additionalfile1PRISMAChecklist.doc

- Additionalfile2searchstrategy.docx

Page 13/13 